\title{
Muscle-inspired ion-sensitive hydrogels with highly tunable mechanical performance for versatile industrial applications
}

\author{
Ping $\mathrm{Li}^{1}$, Ziang Wang ${ }^{1}$, Xinxing $\mathrm{Lin}^{1}$, Xiaolin Wang ${ }^{2 *}$ and Hui Guo ${ }^{1^{*}}$
}

\begin{abstract}
Human muscles are notably toughened or softened with specific inorganic ions. Inspired by this phenomenon, herein we report a simple strategy to endow hydrogels with comparable ion-responsive mechanical properties by treating the gels with different ionic solutions. Semi-crystalline poly(vinyl alcohol) hydrogels are chosen as examples to illustrate this concept. Similar to muscles, the mechanical property of hydrogels demonstrates strong dependence on both the nature and concentration of inorganic ions. Immersed at the same salt concentration, the hydrogels treated with different ionic solutions manifest a broad-range tunability in rigidity (Young's modulus from 0.16 to $9.6 \mathrm{MPa}$ ), extensibility (elongation ratio from $100 \%$ to $570 \%$ ), and toughness (fracture work from 0.82 to $35 \mathrm{MJ} \mathrm{m}^{-3}$ ). The mechanical property well follows the Hofmeister series, where the "salting-out" salts (kosmotropes) have a more pronounced effect on the reinforcement of the hydrogels. Besides, the hydrogels' mechanical performance exhibits a positive correlation with the salt concentration. Furthermore, it is revealed both the polymer solubility from amorphous domains and polymer crystallinity from crystalline domains are significantly influenced by the ions, which synergistically contribute to the salt-responsive mechanical performance. Benefitting from this feature, the hydrogels have demonstrated promising industrial applications, including tunable tough engineering soft materials, anti-icing coatings, and soft electronic devices.
\end{abstract}

Keywords: crystalline hydrogels, ion-responsive, Hofmeister effect, mechanical performance, conductive hydrogels

\section{INTRODUCTION}

Materials with ion-responsive properties are common in living organisms. For instance, the transportation of specific ions across muscle fibers modifies the mechanical performance of our muscles, and therefore leads to the movements of our body. In this process, different ions demonstrate distinct biological effects: the physiological effects of sodium and potassium are poles apart in tuning the motion of our smooth muscle [1]. Inspired by bio-tissues in nature, scientists are keen to conceive man-made engineering materials with similar ion-responsive features in the past few years [2].

Hydrogels are among the most intensively investigated artificial materials for this purpose. As a three-dimensional cross- linked network infiltrated with a large amount of water or biological fluids, hydrogel demonstrates various similarities to biological tissues, such as hydrophilicity, biocompatibility, and flexibility. During the last two decades, hydrogels have embraced increasing interest in numerous engineering [3] and biological applications [4-5]. In particular, stimuli-responsive hydrogels have been vigorously investigated owing to their automatic response to environmental changes [6-8]. Among these smart soft materials, considerable attention is given to ion-responsive hydrogels, especially the hydrogels whose mechanical performance can be finely modified with ions [9].

Admittedly, the ions can play versatile functionalities in regulating hydrogels' mechanical performance, including rigidity, extensibility, and toughness [10]. On the one hand, the strength of coordinate bonds in hydrogel polymer networks is often sensitive to environmental ions. As a typical example, the introduction of appropriate cations (e.g., $\mathrm{Fe}^{3+}$ [11], $\mathrm{Zr}^{4+}$ [12], $\mathrm{Cu}^{2+}[13]$, and $\left.\mathrm{Ca}^{2+}[14]\right)$ inside polyacrylic acid-based hydrogels often leads to the formation of new crosslinking points between metal ions and ligands, and therefore modifies the mechanical performance. However, this toughening process induced by the formation of strong metal-coordinate bonds is often limited to specific pairs between ions and polymers. On the other hand, ions can strikingly decrease the water content of a polyelectrolyte hydrogel by controlling osmotic pressure. Once the gel is shrunk, the polymer volume fraction is enhanced, free volume in the polymer network is compacted, and the material is toughened. This phenomenon has been particularly investigated in recent ionic gels with additional non-covalent associations [15]. However, similar to the previous mentioned mechanism, this strategy proves to be effective only in specific gels. Different from the aforementioned mechanisms, the mechanical performance of hydrogel can also be readily modified by affecting the polymer/water interactions, which is often termed as Hofmeister effect [16]. Hofmeister effect is a qualitative performance ordering of ions based originally on their propensity to salt-out proteins from aqueous solutions. Upon the introduction of ions, hydrogen bonding, dispersion interactions, hydration forces, or even dissolved gas may contribute to this effect by adjusting the affinity of polymers to water [17-19]. Ions can be divided into "kosmotropes" and "chaotropes" with so-called "salting-out" and "salting-in" effects, respectively, indicating how the interactions of small solute molecules with water affect the solubility of macromolecules [20]. The overall order of the anion series is $\mathrm{SO}_{4}{ }^{2-}>\mathrm{CO}_{3}{ }^{2-}>\mathrm{F}^{-}>\mathrm{Ac}^{-}>\mathrm{Cl}^{-}>\mathrm{Br}^{-}>\mathrm{NO}_{3}^{-}>\mathrm{I}^{-}>\mathrm{ClO}_{4}^{-}>$

\footnotetext{
${ }^{1}$ School of Chemical Engineering and Technology, Sun Yat-sen University, Zhuhai 519082, China

${ }^{2}$ School of Pharmacy and State Key Laboratory of Quality Research in Chinese Medicine, Macau University of Science and Technology, Taipa, Macao, China

* Corresponding authors (emails: xilwang@must.edu.mo (Wang X); guoh37@mail.sysu.edu.cn (Guo H))
} 
$\mathrm{SCN}^{-}$and for cations it generally follow the sequence of $\mathrm{K}^{+} \sim$ $\mathrm{Na}^{+}>\mathrm{Li}^{+}>\mathrm{Mg}^{2+}>\mathrm{Ca}^{2+}$ [21]. The salting-out effect tends to enhance the polymer volume fraction [22], strengthen specific physical interactions [23], and consequently reinforce the mechanical behavior of hydrogels, while the salting-in effect has the opposite effect [24]. Compared with the previous two mechanisms, this strategy has better universality, as the solubility of most polymers is largely dependent on the ionic environment. As a typical example, He et al. [25] reported a facile approach to fabricate tough and ductile gelatin hydrogels simply by soaking a virgin gelatin gel in an ammonium sulfate solution. The Young's modulus, fracture strain, and fracture stress demonstrate evident concentration dependence of salt. The authors attributed the high ductility and strength of the hydrogels in salt to the induced enhancement of the chain bundling and hydrophobic interactions. Similarly, the mechanical performances of whey protein isolate hydrogels treated with various salt solutions have been investigated [26]. The infiltration of kosmotropic anions yields stiffer hydrogels, whereas the chaotropic anions soften the hydrogels. Different salts may have significant differences in water binding ability, therefore giving rise to the variation in mechanical performance. Yet, this Hofmeister effect on the ion-responsive mechanical performance of hydrogels has not received sufficient attention until recent years.

In this work, we report a highly tunable mechanical performance with semi-crystalline poly(vinyl alcohol) (PVA) hydrogels inspired by our muscles (Fig. 1a, b). By simply immersing the as-prepared polymer films in different ionic solutions, the resulting hydrogels demonstrate wide-range tunability in rigidity
(Young's modulus from 0.1 to $9.6 \mathrm{MPa}$ ), extensibility (elongation ratio from $100 \%$ to $570 \%$ ), and toughness (fracture work from 0.82 to $35 \mathrm{MJ} \mathrm{m}^{-3}$ ). The mechanical property well follows the Hofmeister series, as the "salting-out" salts (kosmotropes) greatly enhance the rigidity and vice versa. Besides, the mechanical property exhibits a positive correlation with the salt concentration. It is further revealed that ion additives not only influence the solubility of non-crystalline polymer chains but also affect polymer crystallinity. Consequently, both factors contribute to the switchable mechanical performance of hydrogels with this facile approach. Owing to the ion-responsive feature, the hydrogels demonstrate promising application potentials, including tough engineering soft materials, anti-icing coatings, and soft electronic devices.

\section{RESULTS AND DISCUSSION}

PVA semi-crystalline hydrogels were prepared using a facile drying-reswelling method with PVA polymers (Fig. 1c). Upon the drying of PVA aqueous solution by heating at $60^{\circ} \mathrm{C}$, welloriented hydroxyl side groups form strong crystallization domains between polymer chains with the loss of water [27]. Macroscopically, liquid polymer solutions turn into stable PVA films. Subsequently, these films were immersed in pure water or ionic solutions for reswelling. During this process, the polymer crystalline domains remain relatively stable, which serves as physical crosslinking points to maintain the materials at solidstate; the amorphous domains mix with solvent owing to their high hydrophilicity, which absorb a large amount of liquid by osmotic pressure. As the rehydration process of PVA films can be highly influenced by the ionic environment, the important
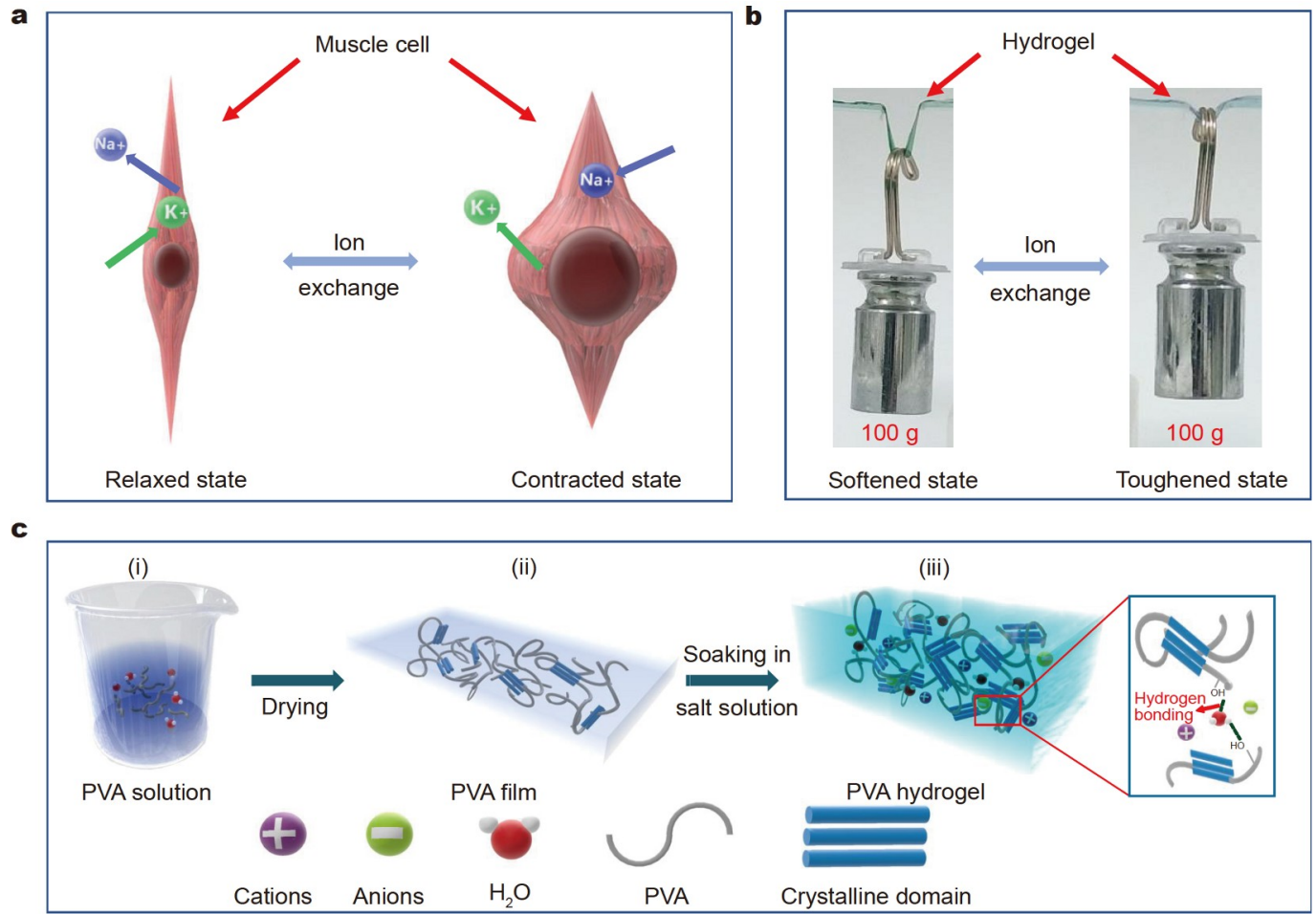

Figure 1 (a) Schematic illustration of a muscle cell with ion-responsiveness. Intracellular potassium ions maintain the muscle cell at a relaxed state. In contrast, muscle cell keeps at a contracted state by ion exchange. (b) By mimicking muscle, PVA hydrogels demonstrate similar ion-responsive mechanical performance. The gel treated with $\mathrm{CrCl}_{3}$ solution (left) becomes significantly reinforced by ion exchange to $\mathrm{Na}_{2} \mathrm{SO}_{4}$ (right). (c) Schematic illustration of the preparation procedure of semi-crystalline PVA hydrogels. PVA solutions (i) were dried at $60^{\circ} \mathrm{C}$, gave rise to PVA films with crystalline domains (ii), and soaked in ionic solutions to form hydrogels (iii). 
role of salt is systematically investigated in the next.

In the first place, we explored the anion effect on the semicrystalline hydrogels' mechanical performance. A series of sodium and potassium solutions were chosen to reswell the PVA films prior to the uniaxial tensile test. As shown in Fig. 2a, PVA hydrogels immersed in different sodium salt solutions presented striking differences in mechanical performance. The sample swollen in $\mathrm{Na}_{2} \mathrm{CO}_{3}$ manifested fracture stress and work of 8.3 $\mathrm{MPa}$ and $35 \mathrm{MJ} \mathrm{m}^{-3}$, respectively, which are around 4 and 10 times higher than that immersed in the same concentrated $\mathrm{NaNO}_{3}$ solution. The distinction is more evident by comparing the elastic modulus. As seen in Fig. 2b, Young's modulus increased from less than $0.6 \mathrm{MPa}$ for gels in pure water, $1.9 \mathrm{MPa}$ in $\mathrm{NaNO}_{3}$, to more than $10 \mathrm{MPa}$ for that in $\mathrm{Na}_{2} \mathrm{SO}_{4}$ solution. In the same manner, potassium salt displayed comparable ionresponsive mechanical performance (Fig. 2c, d). For instance, the sample soaked in $1 \mathrm{~mol} \mathrm{~L}^{-1} \mathrm{KCl}$ solution exhibited rather high fracture stress $(7.3 \mathrm{MPa})$ and strain $(440 \%)$. In contrast, the same material treated with $1 \mathrm{molL}^{-1} \mathrm{KSCN}$ solution only showed ultimate stress of less than $1 \mathrm{MPa}$ with an elongation of about $140 \%$.

According to classic rubbery elasticity [28], Young's modulus of hydrogels may be the most convenient criterion to characterize the reinforcement of hydrogels. Based on these values shown in Fig. 2b, d, the reinforce effect of anion follows the series of $\mathrm{SO}_{4}{ }^{2-}>\mathrm{CO}_{3}{ }^{2-}>\mathrm{Cl}^{-}>\mathrm{H}_{2} \mathrm{PO}_{4}{ }^{-}>\mathrm{NO}_{3}{ }^{-}$for sodium salts and $\mathrm{Cl}^{-}>\mathrm{Br}^{-}>\mathrm{I}^{-}>\mathrm{SCN}^{-}$for potassium salts. Both sequences are in good agreement with previous reports on the solubility of macromolecules, where $\mathrm{SO}_{4}{ }^{2-}, \mathrm{CO}_{3}{ }^{2-}$, and $\mathrm{Cl}^{-}$are typically considered as strong kosmotropes with salting-out effect, whereas $\mathrm{I}^{-}$and $\mathrm{SCN}^{-}$are normally deemed as classic chaotropes. Interestingly, the samples swollen in most kosmotropic environments manifested an obvious strain-softening at smaller strain and strain-hardening performance under large deformation, while the one with the opposite type of salts often demonstrated a classical tensile mode. This phenomenon can be ascribed to the enhancement of hydrogels' interchain physical interactions in the kosmotropic solutions. The uniaxial tensile test leads to the breakage of such secondary forces at relatively small strains, which gives rise to the strain-softening performances. In contrast, the large-scale deformation leads to the total straightening of polymer chains, resulting in the strain-hardening properties [29]. As plotted in Fig. S1, the tensile behavior significantly deviates from the purely entropic elasticity prediction and beyond $50 \%$ of strain for the sample in $\mathrm{KCl}$, which resembles the results of physical interaction-reinforced hydrogels [30].

After a full comparison between anions, attention is given to cations in the following study. Ten types of chloride salts with the same concentration were fully analyzed and the gels showed entirely different tensile performances concerning their rigidity, extensibility, and toughness. As shown in Fig. 3a, b, hydrogels soaked in strong "salt-out" electrolytes, such as $\mathrm{NaCl}$ and $\mathrm{KCl}$,
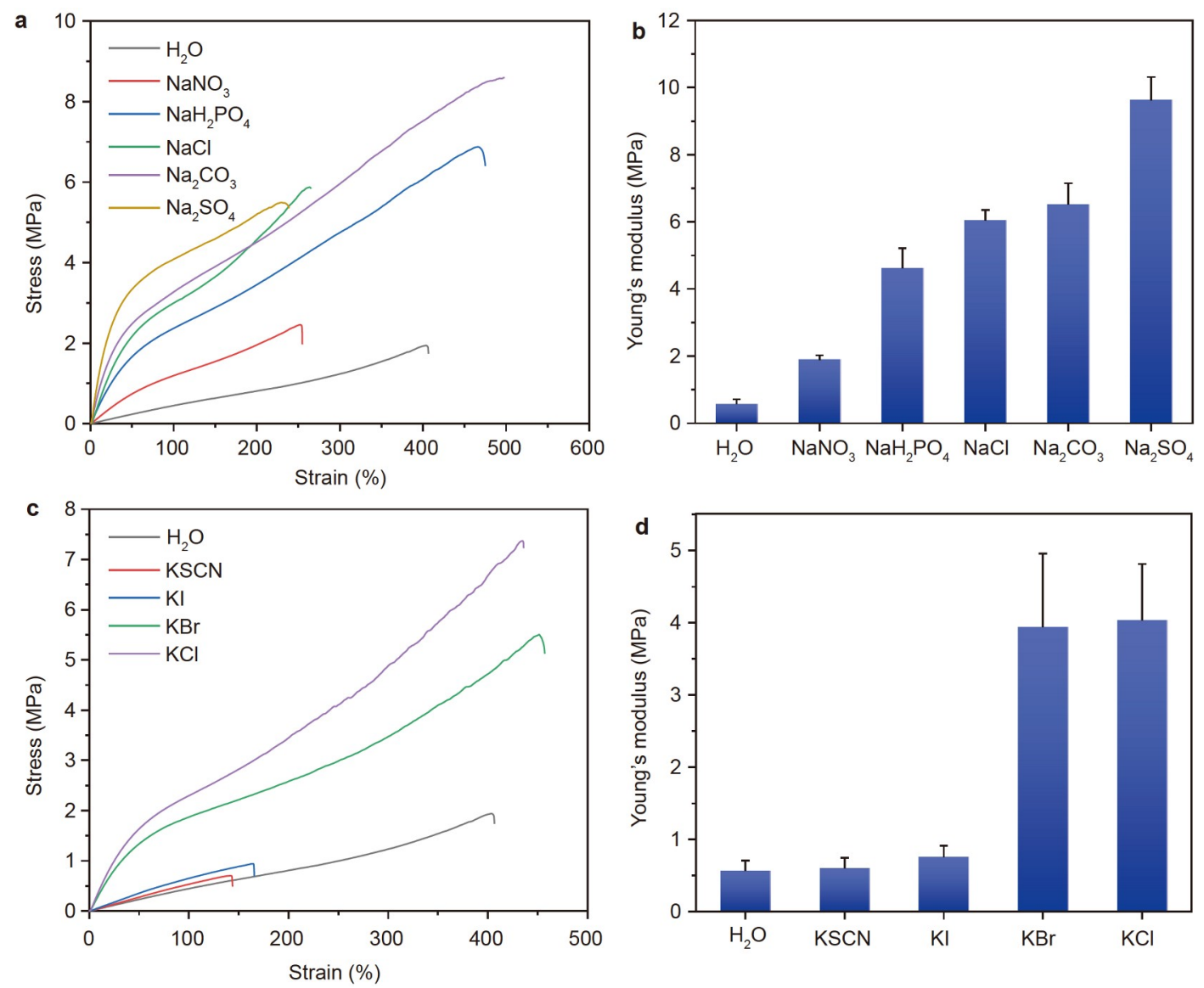

Figure 2 Influence of the anion on the mechanical performance of hydrogels. (a) Uniaxial tensile stress-strain curves of semi-crystalline PVA hydrogels treated with different sodium salt solutions. The Young's modulus is depicted in (b). (c) Uniaxial tensile stress-strain curves of semi-crystalline PVA hydrogels treated with different potassium salt solutions. The corresponding Young's modulus is depicted in (d). Salt concentration was fixed at 1 mol $\mathrm{L}^{-1}$ for all the samples. 

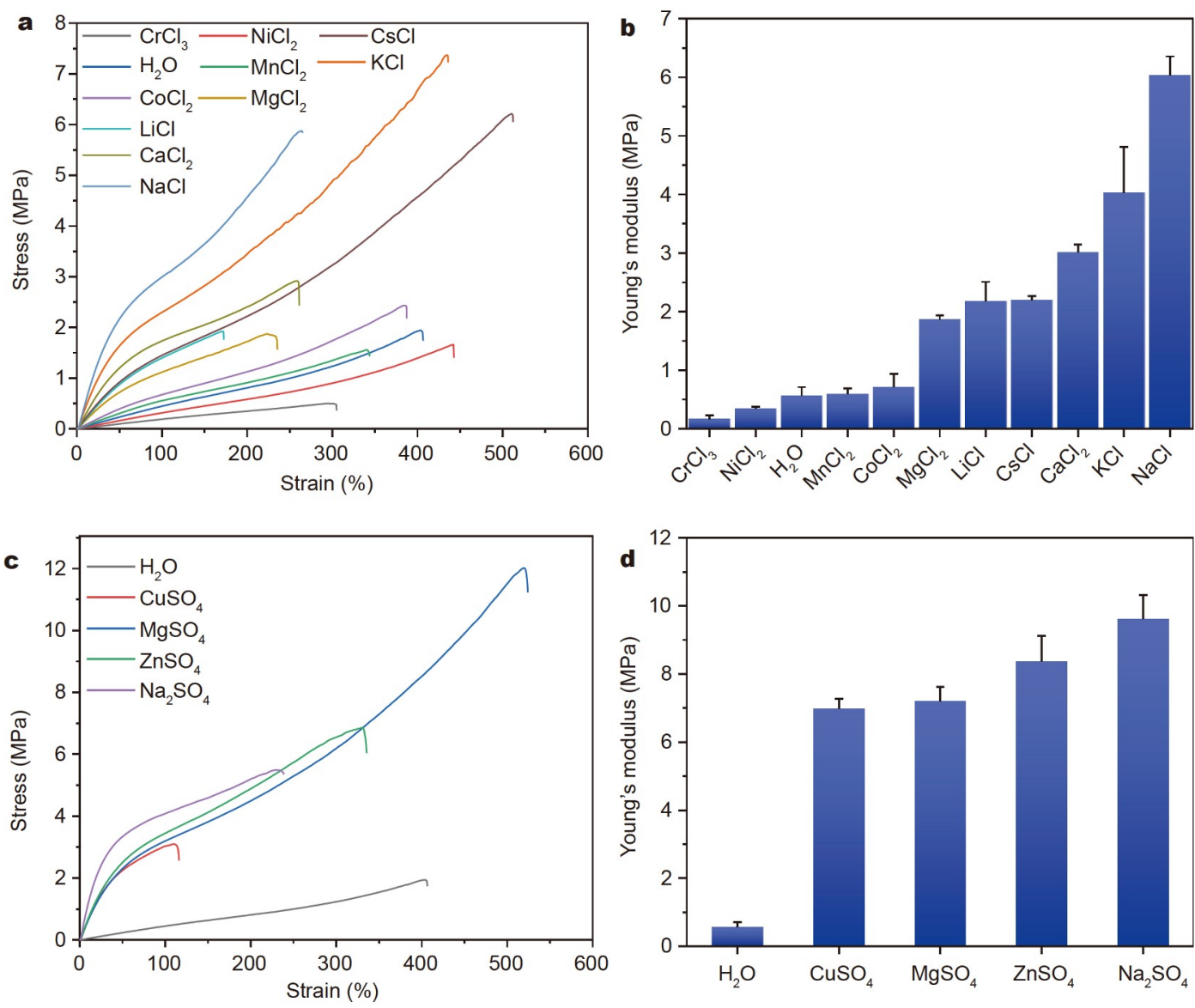

Figure 3 Influence of the cation on the mechanical performance of hydrogels. (a) Uniaxial tensile stress-strain curves of semi-crystalline PVA hydrogels treated with different chloride salt solutions. The Young's modulus is depicted in (b). (c) Uniaxial tensile stress-strain curves of semi-crystalline PVA hydrogels treated with different sulfate salt solutions. The corresponding Young's modulus is depicted in (d). Salt concentration was fixed at 1 mol $\mathrm{L}^{-1}$ for all the samples.

manifested the most excellent tensile performance, with fracture stress as high as 6-8 MPa. In comparison, the ultimate stress was only around $2 \mathrm{MPa}$ for those with low abilities to influence polymer solubility, including $\mathrm{MgCl}_{2}, \mathrm{LiCl}$, and $\mathrm{CsCl}$ solutions. It is further revealed that ionic solutions of transition metals, e.g., $\mathrm{CrCl}_{3}, \mathrm{NiCl}_{2}, \mathrm{CoCl}_{2}$, and $\mathrm{MnCl}_{2}$, which have been rarely investigated in the Hofmeister series [31], have the weakest impact on the reinforcement of PVA semi-crystalline hydrogels. As a typical example, the sample immersed in $\mathrm{CrCl}_{3}$ solution manifested the lowest rigidity and toughness with Young's modulus $\sim 0.16 \mathrm{MPa}$ and work of $0.82 \mathrm{MJ} \mathrm{m}^{-3}$, which is around 40 and 10 times lower than that in $\mathrm{NaCl}$ media. In parallel, cations also exhibited a certain level of influence with sulfate salts (Fig. 3c, d), where stronger kosmotropes have better reinforcement performance. However, the difference is less prominent than that for chloride salts, which may be attributed to leveling effect of the strong "salting-out" effect induced by the sulfate anion. Indeed, the role played by cations often overwhelms that by anions in such Hofmeister effect from the previous report [21]. According to these results, an evident sequence appears: $\mathrm{Cr}^{3+}<\mathrm{Ni}^{2+}<\mathrm{Mn}^{2+}$ $<\mathrm{Co}^{2+}<\mathrm{Mg}^{2+}<\mathrm{Li}^{+} \sim \mathrm{Cs}^{+}<\mathrm{Ca}^{2+}<\mathrm{K}^{+}<\mathrm{Na}^{+}$, which is globally in well coincidence with intensively investigated Hofmeister series.

From the previous performance, it is clear that the mechanical performance of hydrogels can be easily modified just by changing the ionic solution in which they are immersed. However, the mechanism of such switchable mechanical performance requires further investigation. When the samples are soaked in different Hofmeister ionic solutions, surely that salt can affect the hydration level of hydrogels, thus modifying the polymer fraction in this soft matter. Indeed, the PVA hydrogels treated with "kosmotropes" display much more compacted morphology than that in "chaotropes", which is in good agreement with the previous report [26] (Fig. S2).

Based on classical rubber elasticity theory [28], the Young's modulus $(E)$ of hydrogels having no permanent entanglements is directly related to the polymer volume fraction, that is:

$E / \varphi=3 v_{\mathrm{e}} R T$,

where $\varphi$ is the volume fraction of polymer, $v_{\mathrm{e}}$ is the elastically effective polymer chain density at the solvent-free state, $R$ is the gas constant, and $T$ is the absolute temperature. Indeed, as shown in Fig. 4a, the elastic modulus of all the samples displayed a positive correlation with the polymer fraction. As a whole, the better the "salting-out" effect, the higher the polymer fraction, and the better mechanical performance. This is well reasonable as the "kosmotropes" decrease the affinity of water for polymer, disturb the hydrogen bonds between the polymer and water in the amorphous regions, decrease the water content, and consequently enhance the rigidity of hydrogels.

Meanwhile, other effects may also contribute to the responsive 

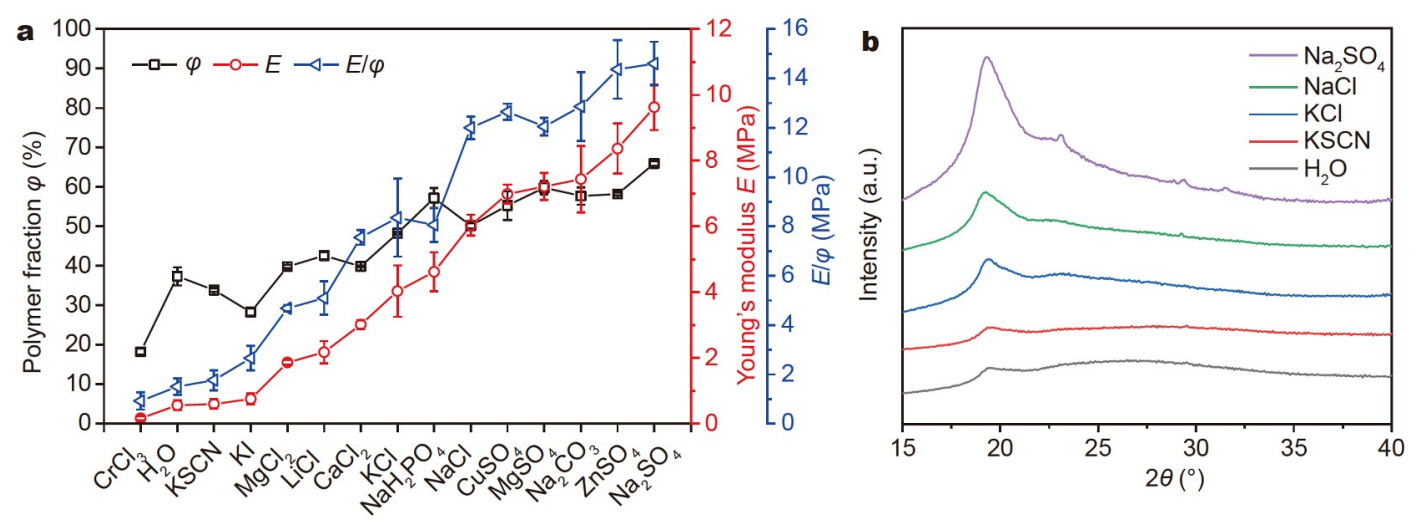

Figure 4 (a) Young's modulus, polymer fraction, normalized modulus $(E / \varphi)$ of semi-crystalline PVA hydrogels treated with different ionic solutions $\left(1 \mathrm{~mol} \mathrm{~L}^{-1}\right)$. (b) XRD spectra of PVA hydrogels soaked in different ionic solutions $\left(1 \mathrm{~mol} \mathrm{~L}^{-1}\right)$. Note that the hydrogels' Young's modulus decrease follows the sequence from the top $\left(\mathrm{Na}_{2} \mathrm{SO}_{4}\right)$ to bottom (water).

mechanical performance. To exclude the polymer fraction's contribution, we normalized the modulus as a function of salt type by plotting the normalized modulus $(E / \varphi)$. According to Equation (1), the $E / \varphi$ should be a constant value $\left(3 v_{\mathrm{e}} R T\right)$ for all the samples if no other factor rules. However, this value keeps unidirectional growth with the strength of the "salting-out" effect, from 0.6 to $14.3 \mathrm{MPa}$. This salt-dependent event clearly indicates that polymer fraction only partly contributes to the ion-responsive toughening of hydrogels. In point of fact, the mechanical performance of PVA hydrogels without crystalline domains presented a much weaker salt dependence (Fig. S3). Inside such semi-crystalline hydrogels, amorphous and crystalline domains coexist. While salt can influence the polymer fraction generally due to the controlling hydration level of materials' amorphous regions, we anticipate that salt may also have an impact on the crystalline domains. To verify this hypothesis, we checked the crystalline zones by X-ray diffraction (XRD) measurement (Fig. 4d). Evidently, salts can significantly affect the crystalline level following the Hofmeister series. Whereas the gel prepared in $\mathrm{Na}_{2} \mathrm{SO}_{4}$ solution displayed a sharp crystalline peak at $18^{\circ}-21^{\circ}$, which is a characteristic peak from PVA crystallization, the ones in KSCN solution or pure water had rather weak signals in the same range. From this point, both crystalline and amorphous regions are strongly influenced by the nature of salt additive, therefore the mechanical properties present clear ion responsiveness.

To further testify this opinion, we subsequently investigated the salt concentration effect. $\mathrm{NaCl}$ was selected as an example due to its strong "salting-out" effect. As depicted in Fig. 5a, the hydrogels' elastic modulus experienced a steady but sharp increase upon raising the concentration of $\mathrm{NaCl}$ from 0 to $1 \mathrm{~mol} \mathrm{~L}^{-1}$. Similar trend is also detected by the cyclic tensile test (Fig. 5b and Fig. S4). The dissipated energy of loading-unloading, which characterizes the energy dissipation and self-recovery ability of materials, displayed strong positive dependence on the salt concentration. At the same time, the residual strain became increasingly evident in more concentrated ionic solutions, which implied an increase in plasticity. With the aid of the previous method in Fig. 4, we also verified that both the polymer faction in non-crystalline domains and crystallinity in crystalline domains are closely related to the salt concentration (Fig. 5c, d).

Benefitting from the ion-responsive mechanical performance, these hydrogels embrace various interests for numerous engi- neering and biological applications. Primarily, the semi-crystalline hydrogels present excellent mechanical performance in kosmotropic salt. As depicted in Fig. 6a, a piece of gel slice prepared in $1 \mathrm{~mol} \mathrm{~L}^{-1} \mathrm{NaCl}$ solution can withstand a weight as high as $2 \mathrm{~kg}$. For a better presentation, we compare our gels with various existing tough hydrogels and bio-tissues. As shown in Fig. 6b, the mechanical performances of our hydrogels treated with ionic solutions are superior to most reported hydrogels and soft tissue both in terms of rigidity and fracture stress. More importantly, these gels demonstrate large-scale regulation of mechanical property by extremely facile methods, which surpass most existing hydrogels. Moreover, the presence of salt ions lowers the melting point of the solvent in hydrogels, thus endowing the materials with satisfactory anti-icing and anti-frost capacity (Fig. 6c). The hydrogel soaked in $\mathrm{NaCl}$ solution shows super-low anti-icing debonding strength of less than $5 \mathrm{kPa}$ under a harsh low temperature, which is superior to the ones with frequently used anti-icing agent dimethyl sulfoxide (DMSO)/ $\mathrm{H}_{2} \mathrm{O}$ [32] and glycerol/ $\mathrm{H}_{2} \mathrm{O}$ [33]. Another interesting feature is related to the high conductivity in gels due to the ionic additives. As shown in the inset photo in Fig. 6d, semi-crystalline PVA hydrogels treated with $1 \mathrm{~mol} \mathrm{~L}^{-1} \mathrm{CaCl}_{2}$ can serve as electric wires to light a red light-emitting diode (LED) bulb in an electrocircuit (the ionic conductivity of hydrogel: $0.68 \mathrm{~S} \mathrm{~m}^{-1}$ ). More importantly, based on the excellent conductivity and flexibility, the PVA hydrogels prepared in ionic solutions can serve as strain sensors for monitoring human motions. As depicted in Fig. 6d and Fig. S5, the salt-incorporated hydrogel demonstrates a rather linear response to strain. The sensor also displays good repeatability in the detection of motions: during the cyclic deformation of $10 \%$ strain for as many as $\sim 100$ cycles, the sensor still generates stable output signals (Fig. 6e). Benefitting from these excellent electrical properties, the hydrogel can be applied as an epidermal sensor to detect the motion of finger bending with good repeatability (Fig. 6d). Absolutely, more promising applications are well foreseeable based on the versatility and tunability of such soft matter.

\section{CONCLUSION}

In summary, we demonstrate a facile strategy to fabricate ionresponsive hydrogels with large-scale tunability in mechanical performance, which resembles human muscles. After the treatment with different ionic solutions, the model system PVA 

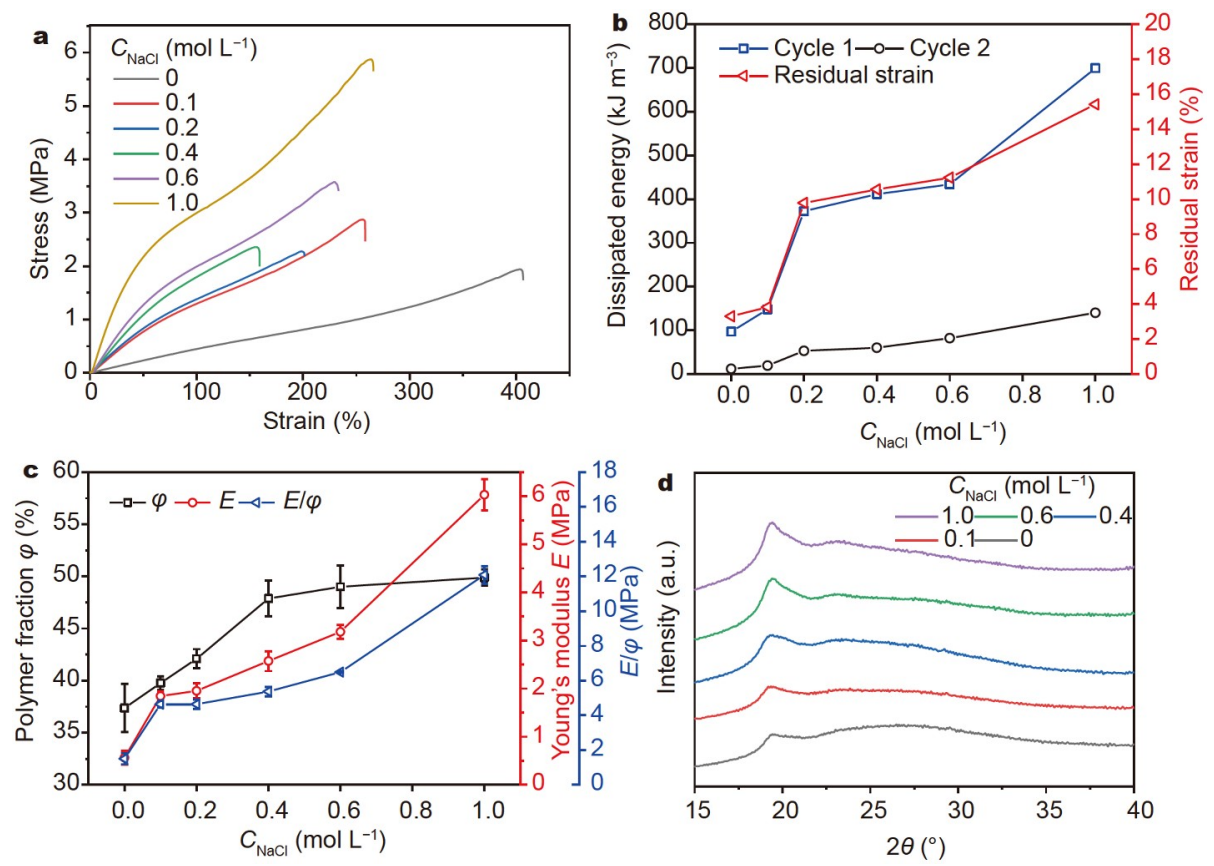

Figure 5 Tensile stress-strain curves of semi-crystalline PVA hydrogels treated with $\mathrm{NaCl}$ at different salt concentrations. (a) Uniaxial tensile stress-strain curves, and (b) cyclic tensile properties of PVA hydrogels with $\mathrm{NaCl}$ treatment at different concentrations. Upon loading-unloading curve at a fixed strain of $100 \%$, the dissipated energy of the first cycle (Cycle 1), the second cycle (Cycle 2), and residual strain of Cycle 2 are presented as a function of NaCl concentration. (c) Young's modulus, polymer fraction, normalized modulus $(E / \varphi)$, and (d) XRD spectra of PVA semi-crystalline hydrogels treated with different concentrated $\mathrm{NaCl}$ solutions ranging from 0 to $1 \mathrm{~mol} \mathrm{~L}^{-1}$.
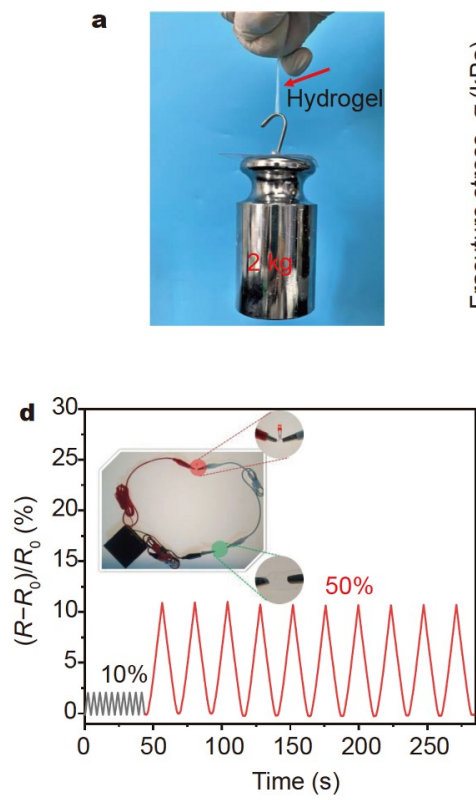
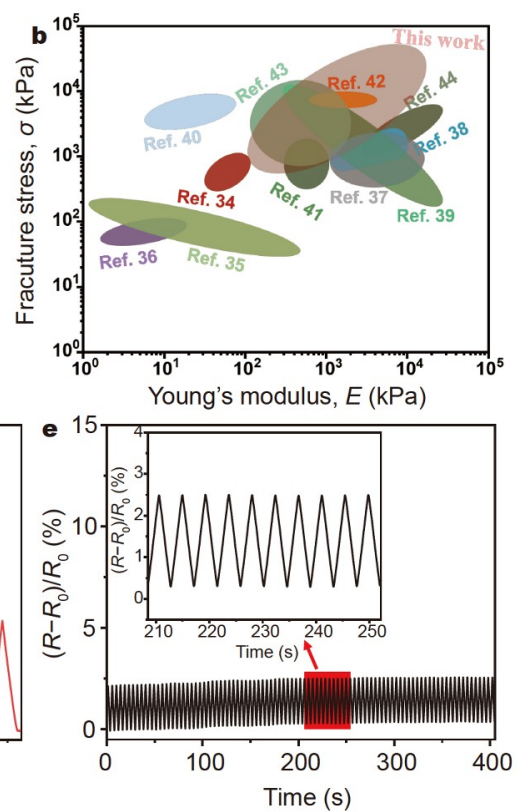
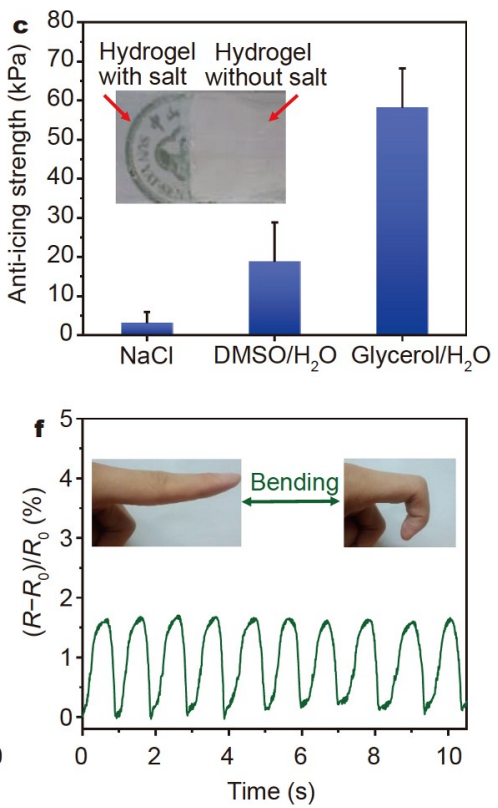

Figure 6 Representative applications of ion-responsive PVA hydrogels. (a) Tough hydrogels. The photo demonstrates that a piece of the hydrogel can withstand weight as high as $2 \mathrm{~kg}$ after soaking in $1 \mathrm{~mol} \mathrm{~L}^{-1} \mathrm{NaCl}$ solution. (b) Mechanical property chart of versatile tough hydrogels with fracture stress versus Young's modulus. Materials include the semi-crystalline PVA hydrogels in this work, anisotropic PVA gel [34], alginate/polyacrylamide gel [35], nanocomposite gel [36], PVA-PAAm gel [37], polyampholytes gel [38], block copolymer double network gel [39], tetra-arm polyethylene glycol gel [40], polyacrylamide- $c o$-methyl acrylate/spiropyran gel [41], $\mathrm{Fe}^{3+}$-poly(acrylamide-co-acrylic acid) gel [42], double network gel [43], and cartilage [44]. (c) Antiicing performance of PVA hydrogels soaked in $25 \mathrm{wt} \% \mathrm{NaCl}$ solution, $\mathrm{DMSO} / \mathrm{H}_{2} \mathrm{O}\left(\right.$ mole ratio $=1: 1$ ), and glycerol/ $\mathrm{H}_{2} \mathrm{O}($ weight ratio $=2: 1)$ at $-10^{\circ} \mathrm{C}$. The inset photo shows the anti-frost performance of PVA hydrogel at $-10^{\circ} \mathrm{C}$ for $10 \mathrm{~min}$ at a relative humidity of $99 \%$ (left: treated with $25 \mathrm{wt} \% \mathrm{NaCl}$ solution; right: without any ionic solution). (d) Time-dependent relative resistance changes of the flexible sensors at different strains (10\% and 50\%). The left inset photo indicates PVA hydrogel soaked in $1 \mathrm{~mol} \mathrm{~L}^{-1} \mathrm{CaCl}_{2}$ solution possessed excellent ionic conductivity when connected to a red LED bulb. The green circle shows the conductive hydrogel, and the red circle shows the red LED bulb. (e) Response of the PVA hydrogels to repeated loading and unloading of $10 \%$ strain for $400 \mathrm{~s}$, showing the good stability of this strain sensor. (f) Real-time response of the sensor to the bending of a forefinger. 
hydrogels experience a programmable change in rigidity, extensibility, and toughness. Both the polymer crystallinity and amorphous polymer solubility of the hydrogels are significantly influenced by salt additives, which contribute to the hydrogels' ion-responsive mechanical properties. In this way, the switchable mechanical performance can be extended to other polymer hydrogels, or even more universally to other external stimuli. This work provides a facile approach to regulate the mechanics of soft matter and will endow ion-responsive hydrogels with more favorable applications in biomedical, agricultural, and industrial fields in the future.

\section{Received 7 April 2021; accepted 28 May 2021; published online 4 August 2021}

1 Borkhvardt V. Muscle contraction: Theory and facts. Bio Comm, 2018, 63: $106-108$

2 Montero de Espinosa L, Meesorn W, Moatsou D, et al. Bioinspired polymer systems with stimuli-responsive mechanical properties. Chem Rev, 2017, 117: 12851-12892

3 Calvert P. Hydrogels for soft machines. Adv Mater, 2009, 21: 743-756

4 Wang X, Ronsin O, Gravez B, et al. Nanostructured dense collagenpolyester composite hydrogels as amphiphilic platforms for drug delivery. Adv Sci, 2021, 8: 2004213

5 Qiu Y, Park K. Environment-sensitive hydrogels for drug delivery. Adv Drug Deliver Rev, 2001, 53: 321-339

6 Guo H, Sanson N, Marcellan A, et al. Thermoresponsive toughening in LCST-type hydrogels: Comparison between semi-interpenetrated and grafted networks. Macromolecules, 2016, 49: 9568-9577

7 Guo H, Nakajima T, Hourdet D, et al. Hydrophobic hydrogels with fruit-like structure and functions. Adv Mater, 2019, 31: 1900702

8 Guo H, Mussault C, Marcellan A, et al. Hydrogels with dual thermoresponsive mechanical performance. Macromol Rapid Commun, 2017, 38: 1700287

9 Dompé M, Cedano-Serrano FJ, Vahdati M, et al. Underwater adhesion of multiresponsive complex coacervates. Adv Mater Interfaces, 2020, 7: 1901785

10 Huang KT, Ishihara K, Huang CJ. Polyelectrolyte and antipolyelectrolyte effects for dual salt-responsive interpenetrating network hydrogels. Biomacromolecules, 2019, 20: 3524-3534

11 Chen Q, Yan X, Zhu L, et al. Improvement of mechanical strength and fatigue resistance of double network hydrogels by ionic coordination interactions. Chem Mater, 2016, 28: 5710-5720

$12 \mathrm{Yu} \mathrm{HC}, \mathrm{Li} \mathrm{CY}, \mathrm{Du} \mathrm{M}$, et al. Improved toughness and stability of $\mathrm{\kappa}-$ carrageenan/polyacrylamide double-network hydrogels by dual crosslinking of the first network. Macromolecules, 2019, 52: 629-638

13 Das Mahapatra R, Imani KBC, Yoon J. Integration of macro-crosslinker and metal coordination: A super stretchable hydrogel with high toughness. ACS Appl Mater Interfaces, 2020, 12: 40786-40793

14 Henderson KJ, Zhou TC, Otim KJ, et al. Ionically cross-linked triblock copolymer hydrogels with high strength. Macromolecules, 2010, 43: 6193-6201

15 Fan $\mathrm{H}$, Wang J, Tao Z, et al. Adjacent cationic-aromatic sequences yield strong electrostatic adhesion of hydrogels in seawater. Nat Commun, 2019, 10: 5127

16 Baldwin RL. How Hofmeister ion interactions affect protein stability. BioPhys J, 1996, 71: 2056-2063

17 Jungwirth P, Cremer PS. Beyond Hofmeister. Nat Chem, 2014, 6: 261263

18 Gurau MC, Lim SM, Castellana ET, et al. On the mechanism of the Hofmeister effect. J Am Chem Soc, 2004, 126: 10522-10523

19 Guo H, de Magalhaes Goncalves M, Ducouret G, et al. Cold and hot gelling of alginate-graft-PNIPAM: A schizophrenic behavior induced by potassium salts. Biomacromolecules, 2018, 19: 576-587

20 Zhang Y, Furyk S, Bergbreiter DE, et al. Specific ion effects on the water solubility of macromolecules: PNIPAM and the Hofmeister series. J Am
Chem Soc, 2005, 127: 14505-14510

21 Yasumoto N, Kasahara N, Sakaki A, et al. Ion-specific swelling behaviors of partially quaternized poly(4-vinyl pyridine) gel. Colloid Polym Sci, 2006, 284: 900-908

22 Tuncaboylu DC, Sari M, Oppermann W, et al. Tough and self-healing hydrogels formed via hydrophobic interactions. Macromolecules, 2011, 44: 4997-5005

23 Sun X, Luo C, Luo F. Preparation and properties of self-healable and conductive PVA-agar hydrogel with ultra-high mechanical strength. Eur Polym J, 2020, 124: 109465

24 Wu S, Hua M, Alsaid Y, et al. Poly(vinyl alcohol) hydrogels with broadrange tunable mechanical properties via the Hofmeister effect. Adv Mater, 2021, 33: 2007829

$25 \mathrm{He} \mathrm{Q}$, Huang $\mathrm{Y}$, Wang S. Hofmeister effect-assisted one step fabrication of ductile and strong gelatin hydrogels. Adv Funct Mater, 2018, 28: 1705069

26 Lin J, Huang Y, Wang S. The Hofmeister effect on protein hydrogels with stranded and particulate microstructures. Colloids Surfs BBiointerfaces, 2020, 196: 111332

27 Otsuka E, Suzuki A. A simple method to obtain a swollen PVA gel crosslinked by hydrogen bonds. J Appl Polym Sci, 2009, 114: 10-16

28 Obukhov SP, Rubinstein M, Colby RH. Network modulus and superelasticity. Macromolecules, 1994, 27: 3191-3198

29 Guo H, Mussault C, Brûlet A, et al. Thermoresponsive toughening in LCST-type hydrogels with opposite topology: From structure to fracture properties. Macromolecules, 2016, 49: 4295-4306

30 Guo H, Sanson N, Hourdet D, et al. Thermoresponsive toughening with crack bifurcation in phase-separated hydrogels under isochoric conditions. Adv Mater, 2016, 28: 5857-5864

31 Demirörs AF, Arslan M, Dag Ö. The effect of anions of transition metal salts on the structure of modified mesostructured silica films and monoliths. Microporous Mesoporous Mater, 2007, 98: 249-257

32 Guo $\mathrm{M}, \mathrm{Wu} \mathrm{Y}$, Xue S, et al. A highly stretchable, ultra-tough, remarkably tolerant, and robust self-healing glycerol-hydrogel for a dualresponsive soft actuator. J Mater Chem A, 2019, 7: 25969-25977

33 Lu C, Chen X. All-temperature flexible supercapacitors enabled by antifreezing and thermally stable hydrogel electrolyte. Nano Lett, 2020, 20: 1907-1914

34 Zhang L, Zhao J, Zhu J, et al. Anisotropic tough poly(vinyl alcohol) hydrogels. Soft Matter, 2012, 8: 10439-10447

35 Sun JY, Zhao X, Illeperuma WRK, et al. Highly stretchable and tough hydrogels. Nature, 2012, 489: 133-136

36 Haraguchi K, Takehisa T. Nanocomposite hydrogels: A unique organicinorganic network structure with extraordinary mechanical, optical, and swelling/de-swelling properties. Adv Mater, 2002, 14: 1120-1124

37 Li J, Suo Z, Vlassak JJ. Stiff, strong, and tough hydrogels with good chemical stability. J Mater Chem B, 2014, 2: 6708-6713

38 Sun TL, Kurokawa T, Kuroda S, et al. Physical hydrogels composed of polyampholytes demonstrate high toughness and viscoelasticity. Nat Mater, 2013, 12: 932-937

39 Zhang HJ, Sun TL, Zhang AK, et al. Tough physical double-network hydrogels based on amphiphilic triblock copolymers. Adv Mater, 2016, 28: $4884-4890$

40 Sakai T, Matsunaga T, Yamamoto Y, et al. Design and fabrication of a high-strength hydrogel with ideally homogeneous network structure from tetrahedron-like macromonomers. Macromolecules, 2008, 41: 5379-5384

41 Chen H, Yang F, Chen Q, et al. A novel design of multi-mechanoresponsive and mechanically strong hydrogels. Adv Mater, 2017, 29: 1606900

42 Lin $\mathrm{P}, \mathrm{Ma} \mathrm{S}$, Wang X, et al. Molecularly engineered dual-crosslinked hydrogel with ultrahigh mechanical strength, toughness, and good selfrecovery. Adv Mater, 2015, 27: 2054-2059

43 Gong JP, Katsuyama Y, Kurokawa T, et al. Double-network hydrogels with extremely high mechanical strength. Adv Mater, 2003, 15: 11551158

44 Little CJ, Bawolin NK, Chen X. Mechanical properties of natural cartilage and tissue-engineered constructs. Tissue Eng Part B-Rev, 2011, 


\section{7: $213-227$}

Acknowledgements This work was supported by the National Natural Science Foundation of China (51903253), the Natural Science Foundation of Guangdong Province of China (2019A1515011150 and 2019A1515011258), and the Science and Technology Development Fund of Macao (FDCT 0083/ 2019/A2).

Author contributions Guo $\mathrm{H}$ and Wang $\mathrm{X}$ designed and supervised the study and revised the manuscript. Li P and Wang Z prepared the hydrogels and characterized their mechanical properties; Li P and Lin X contributed to the application of the hydrogels and the data analysis. Li P and Guo $\mathrm{H}$ wrote the manuscript. All the authors contributed to the general discussion.

Conflict of interest The authors declare that they have no conflict of interest.

Supplementary information Experimental details and supporting data are available in the online version of the paper.

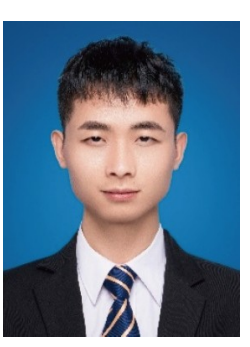

Ping Li is currently a master student at Sun Yat-sen University. His research interest mainly focuses on the development and applications of high-toughness hydrogel materials.

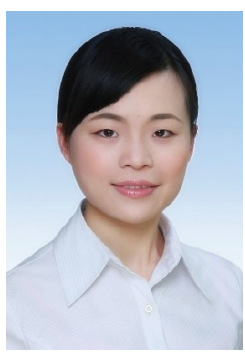

Xiaolin Wang obtained her PhD degree in 2015 from Sorbonne Université, Paris, France and worked for two more years in the same lab for her postdoctoral research. She joined the School of Pharmacy and State Key Laboratory of Quality Research in Chinese Medicines at Macau University of Science and Technology (MUST) as an assistant professor in 2018. Her current research interests are focused on the nano/microparticles and hydrogel-based drug delivery systems in the field of wound healing and cardiovascular diseases.

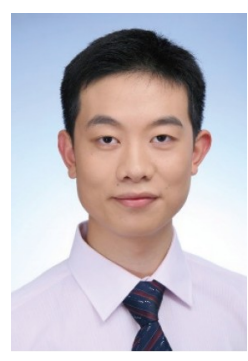

Hui Guo is an associate professor at Sun Yat-sen University, China. He graduated from Shanghai Jiao Tong University, China in 2012 and Universite Pierre et Marie Curie, France in 2015. Then he was engaged in two periods of postdoctoral research under the supervision of professors Dominique Hourdet and Jian Ping Gong before back to China. His current research interests include smart hydrogels, functional soft matters, and hydrogel-based biomedical applications.

\section{受肌肉启发的离子响应韧性可调型水凝胶的开发与 应用}

李平 ${ }^{1}$, 王子昂 ${ }^{1}$, 林新星 ${ }^{1}$, 王晓琳 ${ }^{2^{*}}$, 郭辉 ${ }^{1^{*}}$

摘要 肌肉组织可因特定离子的刺激而发生显著机械性能的变化. 受 此现象启发, 本文通过一种简单高效的策略, 赋予结晶型聚乙烯醇水凝 胶显著的盐响应性特征. 与肌肉组织类似, 这类水凝胶的机械性能展现 出强烈的离子种类及浓度的依赖关系. 经过相关浓度盐溶液处理后, 该 水凝胶在硬度 (杨氏模量 0.16-9.6 MPa) 、延展性(伸长率 100\%$570 \%$ )和㓞性 (断裂功 0.82-35 $\mathrm{MJ} \mathrm{m}^{-3}$ ) 方面表现出大范围的可调控性. 其机械性能的变化很好地遵循了霍夫迈斯特序列, 即强盐析性质的离 子(kosmotropes)在水凝胶增韧中彰明较著. 同时, 该水凝胶的机械性 能与盐溶液浓度表现出显著的正相关性. 实验证明, 离子可显著影响非 晶区的高分子水化程度和结晶区的聚合物结晶度, 进而协同调控水凝 胶的机械性能. 得益于此, 这类水凝胶在韧性可调工程软材料、防冰涂 料和柔性电子设备等领域展现出良好的应用前景. 